\section{Research Square}

Preprints are preliminary reports that have not undergone peer review.

They should not be considered conclusive, used to inform clinical practice, or referenced by the media as validated information.

\title{
Genome Wide Pleiotropic Analysis to Identify Novel Variants and Improve Genetic Risk Score Construction
}

\section{Xiaofeng Zhu ( $\nabla$ xxz10@case.edu )}

Case Western Reserve University https://orcid.org/0000-0003-0037-411X

Luke Zhu

2. Center for Human Genetics \& Genomics, Department of Medicine, New York University Langone Health

Heming Wang

Brigham and Women's Hospital https://orcid.org/0000-0002-1486-7495

Richard Cooper

Loyola University Stritch School of Medicine

Aravinda Chakravarti

2. Center for Human Genetics \& Genomics, Department of Medicine, New York University Langone Health https://orcid.org/0000-0002-4264-2285

Article

Keywords: blood pressure, Mendelian Randomization, GWAS pleiotropy analysis

Posted Date: March 10th, 2021

DOI: https://doi.org/10.21203/rs.3.rs-133000/v2

License: @ (1) This work is licensed under a Creative Commons Attribution 4.0 International License. Read Full License 


\section{Abstract}

Systolic and diastolic blood pressure (S/DBP) are highly correlated and modifiable risk factors for cardiovascular disease (CVD). We report here a bidirectional Mendelian Randomization (MR) and GWAS pleiotropy analysis of S/DBP summary statistics from large published BP GWAS and construct a composite genetic risk score (GRS), capturing respectively $21 \%, 11 \%$, and $227 \%$ more of SBP, DBP and PP heritability than achieved with the traditional GRS The composite GRS improves the prediction of hypertension and CVD in persons of European as well as African and Asian descent. We identified and confirmed 120 novel BP pleiotropic variants that are not in linkage disequilibrium with known variants, including 17 novel BP loci. We further observed significant age-modulated genetic effects on BP, hypertension and CVD in both Europeans and Asians. Our study provides further insight into BP regulation and provides a novel way to construct a GRS for correlated traits.

\section{Introduction}

Poorly controlled blood pressure (BP) accounts for a large portion of the excess risk for cardiovascular disease (CVD), stroke, and heart failure ${ }^{1}$. Understanding biological mechanisms for BP regulation could potentially help improve BP control and lead to a reduction in the burden of CVD. BP is characterized as systolic and diastolic blood pressure (SBP/DBP) and these measurements are long-standing risk predictors for CVD. Genome-wide association studies (GWAS) have to date been performed on BP traits separately by focusing on main effects. In studies that included subjects of diverse ancestry over 1,000 BP-associated loci have been identified ${ }^{2-14}$. Genome-wide search of gene-environment interactions on BP traits have been recently conducted, however limited new associations have been identified, in part because of low statistical power ${ }^{13 ;} 14$. Although many variants are shared among SBP and DBP as correlated traits, a portion of the variants are associated only with SBP or DBP separately, suggesting among the true positive associations there may be evidence of trait-specific biological mechanisms. It has also been reported that a joint analysis of SBP and DBP leads to the identification of BP variants that are missed by analyzing SBP or DBP separately ${ }^{9}$. However prior studies have not addressed the mechanism underlying the relationship of SBP and DBP, which may reflect arterial stiffness or arterial compliance ${ }^{15}$. Dissecting the causal relationships of BP variants on SBP and DBP, in particular, whether the variants affect SBP and DBP through the same (mediation) or different (pleiotropic) paths, and how many pleiotropic variants contribute jointly to these highly correlated traits, may advance our understanding of the biology of BP regulation.

Genetic risk scores (GRS) are usually constructed as linear combinations of individual variant effects estimated from GWAS to predict individual-level risk of common disease. For BP, a final GRS is the average of SBP- and DBP-specific GRS ${ }^{3 ;} 6$ and the GRS predicts the risk of CVD. However, published BP GRS's have explained no more than $6 \%$ of the heritability, and have limited predictive power for HTN and CVD. GWAS of gene-age interaction analysis have identified genetic variants with age-dependent effect sizes, including BP ${ }^{16 ; 17}$, lipid levels ${ }^{18}$ and $\mathrm{BMI}^{19}$. A recent study based on a proportional hazards model reported age-varying risk profiles in nine diseases, including $\mathrm{HTN}^{20}$. However, these studies were under powered because the interactive contribution of a variant by age is often weak.

In this study we address the underlying mechanistic relationship between SBP and DBP by performing a bidirectional Mendelian Randomization (MR) ${ }^{21}$ and GWAS pleiotropy analysis using the summary statistics from $>750,000$ subjects of European ancestry from the UKB and ICBP consortium ${ }^{6}$, followed by the summary statistics of 318,891 subjects of European ancestry from the Million Veteran Program (MVP) ${ }^{12}$. We searched for novel BP variants with pleiotropic effects and constructed a composite GRS using variants with and without pleiotropic effects, and studied the age-varying effects of GRS on prediction of BP, HTN and CVD in groups of European, African and Asian descent.

\section{Results}

We present a bi-directional MR analysis of SBP and DBP using the 1,125 and 1,183 independent genome-wide significant variants for SBP and DBP ( $<$ < $5 \times 10$ ${ }^{8}$ ) as genetic instrumental variables (IVs) obtained from the UKB-ICBP GWAS ${ }^{6}$ and software IMRP22 and MRmix ${ }^{23}$ (Online Methods). We standardized SBP and DBP and obtained an identical causal effect of SBP on DBP and DBP on SBP $(0.864 \pm 0.005$ and $0.862 \pm 0.005$ by IMRP, respectively, Supplementary Table 1), which is significantly larger than the observed trait correlation 0.738 between SBP and DBP in UKB European descents, with estimated $74.8 \%$ of variation is the shared causal contribution between SBP and DBP. The causal estimates by MRmix were concordant $(0.89 \pm 0.012$ and $0.90 \pm 0.01$, respectively, Supplementary Table 1). Among the genetic IVs, $43 \%$ of the variants had pleiotropic effects on SBP and DBP.

We next extended the pleiotropic effect analysis to search for variants with evidence of pleiotropy for SBP and DBP across the whole genome (OnlineMethods). The Manhattan and QQ plots for testing pleiotropy are presented in Fig 1. LD score regression analysis ${ }^{24}$ estimated that $8.7 \%$ of the heritability was due to pleiotropic variants. We observed 906 independent variants $\left(r^{2}<0.05\right)$ reaching genome-wide significance level in pleiotropy tests $\left(\mathrm{P}<5 \times 10^{-8}\right), 234$ of which had not been detected by the univariate GWAS analysis of SBP, DBP or PP in the original UK Biobank+ICBP consortium ${ }^{6}$ (Supplementary Table 2). Among the 234 variants, 201 variants were novel and were not reported in any previous BP GWAS. In the set of associations, 163 variants in 124 loci were within a $1 \mathrm{Mb}$ region of previous reported known BP loci but were not in LD with known BP variants $\left(r^{2}<0.05\right)$; the remaining 38 variants were $1 \mathrm{Mb}$ away from the previous reported known BP loci, and resided in 35 loci; the corresponding locus zoom plots are presented in Supplementary Figure 1. We evaluated the associations of our sentinel SNPs at the 35 novel loci with other traits and disease using GWAS Catalog ${ }^{25}$ and FUMA ${ }^{26}$. The GWAS Catalog and FUMA search of published GWAS showed that 29 of the 35 novel loci are also significantly associated with other traits, including lipid levels, cardiovascular-related outcomes, anthropometric traits, sleep traits, educational attainment, smoking, blood protein level and Schizophrenia (Supplementary Table 3).

Replication of novel signals in Million Veteran Program (MVP). Using the causal effect estimate between SBP and DBP from the UKB-ICBP summary statistics, we performed pleiotropy tests among the 201 novel variants. Eighteen variants in the 35 novel loci (Table 1), and 102 of 163 novel variants on known 124 loci 
could be replicated in MVP at $\mathrm{P}<0.05$ (Supplementary Table 2), suggesting the identified novel pleiotropic signals are not false positives.

Functional analysis. We performed expression quantitative trait locus (eQTL) analysis using GTEx data. Among the 35 novel loci, we identified 26 novel loci with expression quantitative trait locus (eQTL) (Supplementary Table 4) and 11 novel loci with Splicing Quantitative Trait Loci (sQTLs) (Supplementary Table 5). The eQTLs were most often enriched in arterial tissues, followed by adipose, heart and nerve tibial tissues. SNP rs17713879 is an eQTL affecting expression of the SH3YL1 and ACP1 genes in 34 tissues and is also a sQTL affecting splicing of these two genes in 50 tissues. SNP rs112500920 is an eQTL affecting expression of several genes, including EFL 1 and $A B 3 B 2$, in multiple tissues, notably adipose and arterial tissues. SNP rs 12478520 is an eQTL affecting expression of multiple genes, including C2orf72, HTR2B, ARMC9 and PSMD1.

We defined mediation variants as those variants associated with SBP or DBP with $\mathrm{P}<5 \times 10^{-8}$ but not significant in a test for pleiotropy, and pleiotropic variants as the variants reaching genome wide significance $\left(\mathrm{P}<5 \times 10^{-8}\right)$ in a pleiotropy test using the UKB-ICBP data. We assessed tissue enrichment of $B P$ loci using DEPICT ${ }^{27}$ at a false discovery rate $(\mathrm{FDR})<5 \%$ but separated mediation from pleiotropic variants in the analysis. There were 1,324 independent mediation variants and 906 independent pleiotropic variants. DEPICT analysis identified enrichment across 42 and 72 tissues and cells using mediation and pleiotropic variants, respectively (Supplementary Table 6). The enriched tissues are highly similar (correlation=0.78) but notable differences were also observed (Supplementary Figure 2a). Enrichment was greatest for arteries in the cardiovascular system for both mediation and pleiotropic variants $(P=2.8 \times$ $10^{-4}$ and $2.41 \times 10^{-11}$, respectively). In general, enrichments observed for mediation variants were also observed for pleiotropic variants, but not vice versa. For example, heart ventricle, heart valves, heart atria and the atrial appendage were enriched for pleiotropic variants $\left(P<1.23 \times 10^{-3}\right)$ but not for mediation variants (Supplementary Table 6). Pathway enrichments for mediation variants and pleiotropic variants were less well correlated (correlation=0.478, Supplementary Figure 2b). Pleiotropic variants were enriched in many molecular pathways that were missed by mediation variants, including oxygen levels, basement membrane, and renal system development $\left(P<2.23 \times 10^{-7}\right.$ for pleiotropic variants, Supplementary Table 7). Evaluation of enriched mouse knockout phenotype terms pointed to the importance of abnormal kidney morphology, impaired wound healing and increased body weight, etc $\left(P<3.28 \times 10^{-8}\right)$ for pleiotropic variants, although these were absent for mediation variants. Protein-protein interaction subnetwork enrichments were also substantially different; using the pleiotropic variants we identified the HSPG2, DDR1, MMP9, COL4A4, TGM2 and COL4A5 subnetworks $\left(P<1.90 \times 10^{-7}\right)$ which were missed relying solely on mediation variants (Supplementary Table 7).

Improved prediction of BP, hypertension and CVD by including pleiotropic variants. It is known that polygenic scores derived from multiple related traits can improve prediction of outcomes ${ }^{28-31}$ but these approaches do not use the pleiotropic variants directly. Here we constructed a traditional BP GRS using all independent 1,616 BP variants from UKB-ICBP. We further constructed a pleiotropic genetic risk score (PGRS) using the 906 pleiotropic variants (Online Methods). We jointly modeled GRS and PGRS adjusting for age, gender, BMI and 10 principal components, and observed PGRS significantly predicted BP traits, as well as risk of HTN and CVD conditional on GRS in all the models (Table 2A and Figure 2) in UKB European ancestry subjects. The variance explained by GRS only was $5.91 \%, 6.09 \%$ and $2.23 \%$ for SBP, DBP, and PP, increased to $7.13 \%, 6.75 \%$ and $7.27 \%$ when including PGRS, respectively, suggesting independent additive predictive power for the PGRS. Interestingly the PGRS had opposite directional effects for SBP and DBP. We observed odds ratios (ORs) 1.64 and 1.15 for individual GRS and PGRS on risk of HTN $\left(P<1 \times 10^{-300}\right)$, respectively. The observed ORs of individual GRS and PGRS for CVD were 1.21 and $1.06\left(P=1.71 \times 10^{-231}\right.$ and $\left.P=2.74 \times 10^{-25}\right)$, respectively, and increased to 1.66 and $1.19\left(P=9.83 \times 10^{-97}\right.$ and $\left.P=1.28 \times 10^{-13}\right)$ when comparing between the upper and lower quantiles of the GRS and PGRS, respectively. However, the odds ratios were further increased to 6.78 and $2.44\left(P<1 \times 10^{-300}\right.$ and $P=1.15 \times$ $10^{-39}$ ) for HTN and CAD, respectively, when comparing the top decile and quintile with bottom decile and quintile of GRS and PGRS (Figure 2). We observed a clear advantage of including PGRS over GRS only (Figure 2). After including the interactions of age and GRS and PGRS the main effects for GRS and PGRS on BP, HTN and CVD were unchanged. However, we observed a significant interaction effect of age and GRS for all BP traits and HTN, but not for CVD. The interaction of age and PGRS significantly contributed to all BP traits, HTN and CVD (P value between $3.0 \times 10^{-2}$ and $7.74 \times 10^{-29}$, Table $2 \mathrm{~A}$ ).

Extension to other ancestries. We examined associations with BP and CVD of the above defined GRS and PGRS in unrelated African (N=7,904) and South Asian $(\mathrm{N}=8,509)$ subjects in the UKB (Table $2 \mathrm{~B}, \mathrm{C})$. Although sample sizes were much smaller than among UKB European subjects, the GRS was significantly associated with SBP, DBP, PP, HTN and CVD in both UKB African- and Asian-descent subjects. PGRS was associated with a $1.21,-0.35$ and 1.56 mmHg higher for SBP, DBP and PP $\left(P=8.34 \times 10^{-8}, 1.09 \times 10^{-2}\right.$ and $\left.2.05 \times 10^{-27}\right)$ in Africans, a 1.25, -0.64 and $1.89 \mathrm{mmHg}$ higher $\left(P=3.21 \times 10^{-10}, 1.69 \times 10^{-8}\right.$ and $1.50 \times 10^{-}$ $\left.{ }^{46}\right)$ in Asians, respectively. The ORs of PGRS for HTN and CVD were significant $\left(P=9.27 \times 10^{-5}\right.$ and $\left.P=2.81 \times 10^{-2}\right)$ in Asians but not in Africans. The effects of PGRS on DBP were also negative as observed in UKB Europeans. Significant interactions of age and PGRS were again observed for BP traits and CVD in UKB Asians (Table 2 C).

Mendelian Randomization of BP on CAD, MI and stroke. We downloaded the published GWAS summary statistics for CAD, MI and stroke and performed MR analysis of BP on CAD, MI and Stroke, using mediation and pleiotropic variants as IVs separately (Online Methods). Both SBP and DBP causally contributed to $\mathrm{CAD}, \mathrm{MI}$ and stroke using mediation as the IVs. The new trait - $\mathrm{BP}_{\text {pleio }}$ - also causally contributed to CAD, MI and stroke using pleiotropic variants (Online

Methods, Table 3). The estimated ORs ranged from 1.66 to 1.85 per SD unit increase in BP on the three outcomes using mediation variants, and ranged from 1.13 to 1.48 using pleiotropic variants. Our analysis identified 10 to $22 \%$ IVs demonstrating pleiotropic effects for BP and the three clinical outcomes (Table 3 ).

\section{Discussion}

Our analysis of the summary statistics derived from over 1 million subjects describe important aspects of the genetic architecture of the two principle highly correlated BP traits. The bi-directional MR analysis of SBP and DBP demonstrated that 1 SD unit increase of SBP leads to 0.86 SD unit increase of DBP, and vice versa, indicating SBP and DBP share $74.8 \%$ of the variation, presumably attributing to common factors and common biological mechanisms. This shared causal contribution is substantially higher than $55.6 \%$ estimated by phenotype correlation analysis between SBP and DBP. Most of the BP variants identified through SBP or DBP univariate association were mediation variants and these variants contribute to the shared causal contribution and would be expected to

Page $3 / 11$ 
be found in either the SBP or DBP GWAS. The pleiotropic variants are associated with the rest of the BP variation, which is conferred through different biological pathways for SBP and DBP. The pleiotropic variants often demonstrated an effect size that was opposite in direction for SBP and DBP and contributed $8.71 \%$ heritability of the new BP trait. The pleiotropy defined trait BP pleio was highly correlated with PP $(\rho \geq 0.66)$ in UKB whites, taken as an indicator of arterial stiffness and considered as an independent risk factor for CVD ${ }^{32}$. Thus, the $\mathrm{BP}_{\text {pleio }}$ can be an independent risk factor for CVD. This is consistent with the finding that SBP, DBP and $\mathrm{BP}_{\text {pleio }}$ all contributed to risk estimation of CAD, Stroke and MI in the MR analysis (Table 3).

In addition to a large number of known genetic variants that contribute to SBP and DBP reported by the original UK Biobank+ICBP consortium ${ }^{6}$, we identified 906 independent variants demonstrating pleiotropy evidence $\left(P<5 \times 10^{-8}\right), 201$ were undetected by the univariate GWAS analysis of SBP, DBP or PP. Replication analysis in the MVP confirmed 120 of the 201 novel variants, including the 17 novel loci.

In addition to the traditional BP GRS calculated in literature ${ }^{3 ;}$, we defined a composite genetic risk score consisting of GRS and PGRS. The joint model of GRS and PGRS suggested both genetic risk scores independently predict BP, HTN and CVD outcomes (Table 2) in the UK Biobank European ancestry subjects. Additionally, including the PGRS led to substantial increments in heritability for BP traits (Table 2 and Figure 2). Although we observed consistent opposite directional effects of PGRS for SBP and DBP in UK Biobank Europeans, Africans and Asians, the prediction of HTN and CVD risk was significantly improved by including PGRS(Figure 2 C and D). The GRS and PGRS defined on European participants both consistently and significantly predict BP, HTN and CVD in UK Biobank Africans and Asians, suggesting that PGRS is able to improve prediction accuracy across ethnic populations. Recent studies have suggested that current GRS models alone have modest improvement of predictive accuracy for CAD ${ }^{33-35}$. The principle outcome of this set of analyses, therefore, demonstrated that adding PGRS significantly improves the prediction model over the GRS alone. This approach of constructing polygenic risk score is conceptually different from the existing approaches using multiple related traits ${ }^{28-31}$ and could be generalized to other diseases by incorporating multiple disease-related traits through pleiotropy analysis.

Our analysis avoided an examination of the interaction of individual variants and age because of insufficient power. We were able to observe the interaction effects of both GRS and PGRS with age in UKB Europeans for SBP, DBP, PP and HTN although the interaction for CVD was only significant for PGRS (Table 2). The age-PGRS interactions were also replicated in Asians despite a substantially smaller sample size. We observed that the interaction contribution to phenotype variation was consistently small (range from $0.1 \%$ to $0.3 \%$ BP heritability in both UKB Europeans and Asians). The negative interaction contributions of both GRS and PGRS on DBP may partially explain the decline of DBP after 60 years older ${ }^{36}$. In comparison, the interaction of age and GRS was positive for SBP, suggesting genetic effect on SBP increases in older individuals. As noted, the GRS interaction effects in UKB Europeans could not be observed in UKB Africans or Asians, likely as a result of small sample sizes. In comparison, the age-modulated interaction of PGRS were observed in both UKB Europeans and Asians, indicating stronger PGRS interaction effects than for the GRS. In our functional analysis, we observed a wider range of BP related tissues and biological pathways for the BP pleiotropic variants than mediation variants, which may imply that the pleiotropic variants are influenced by a wider range of environmental factors and therefore continue to make an evolving genetic contribution over the age span. Our study also supports an omnigenic model for complex traits ${ }^{37-39}$. In fact, it could be inferred that a pleiotropic variant acts on multiple peripheral genes to impact the expression of a core gene in different biological pathways. As a result, the pleiotropic variants have weak effects on a phenotype and are more difficult to detect in a traditional BP GWAS that focuses on single trait analysis, as we observed in the current analysis. In comparison, mediation variants may be more likely to occur in core genes, although it is clearly important to detect the putative set of core genes as well. We acknowledge that the data presented here can only provide suggestive evidence for that hypothesis.

In conclusion, the new findings we bring forward here include the 906 independent BP pleiotropic variants - 201 of which were previously not identified in traditional BP GWAS - and a novel way to construct polygenic risk score represent a substantial advance in understanding the genetic architecture of highly correlated SBP and DBP.

\section{Online Methods}

Summary statistics of UK Biobank (UKB) and International Consortium for Blood Pressure (ICBP). UKB consists of 458,577 UK European and ICBP consists of 299,024 European decent subjects. GWAS of BP traits were conducted in UKB and ICBP separately and results were meta-analyzed. ${ }^{3 ;} 6$ Our analysis was based on the summary results from the UKB and ICBP GWAS that were calculated based on up to 757,601 participants and $~ 7.1 \mathrm{M}$ genotyped and imputed SNPs with $\mathrm{MAF} \geq 1 \%$ for variants present in both the UKB data and ICBP meta-analysis for SBP, DBP and PP.

Summary statistics of the Million Veteran Program (MVP). The summary statistics of the Million Veteran Program (MVP) consists of 318,891 European ancestry participants and 18.2M genotyped and imputed SNPs for SBP, DBP and PP. ${ }^{12}$ The MVP summary statistics were used for replication analysis.

UKB phenotypic data. (There was an inadvertent mistake in the last version). We analyzed three BP traits in UKB: SBP, DBP and pulse pressure (PP) (SBPDBP). We calculated the mean SBP and DBP values from two baseline BP measurements. We added 15 and $10 \mathrm{mmHg}$ to SBP and DBP for individuals who took antihypertensive medications. Hypertensive cases were defined as either $\mathrm{SBP}>=140$ or $\mathrm{DBP}>=90$ or taking antihypertensive medications. CVD cases in UKB were defined using self-reported baseline information on CVD prevalence and the ICD9 and ICD10 diagnostic codes on hospital admissions. The CVD cases includes ICD10 codes (I210, I211, I212, I213, I214, I219, I21X, I220, I221, I228, I229, I230, I231, I232, I233, 234, I235, I236, I238, I240, I241, I248, I249, I250, I251, I252, I253, I254, I255, I256, I258, I259), and ICD9 codes ("4109", "4119","4129", "4139", "4140", "4141", "4148", "4149"). This procedure resulted 35,968 CVD cases in European, African and Asian ancestries.

Mendelian Randomization analysis.We applied the iterative Mendelian randomization and pleiotropy analysis (IMRP) ${ }^{22}$ and MR mixture model (MRmix) ${ }^{23}$ for bi-directional MR analysis of SBP and DBP, as well as to estimate the causal contributions ofBP on CAD, MI and STROK. IMRP is an iterative approach by 
combining the pleiotropy test and the MR analysis. The iteration starts by performing MR-Egger analysis ${ }^{40}$ to estimate causal effect of an exposure to outcome, following by inverse variance weighted (IVW) ${ }^{41 ; 42}$ analysis until the causal effect estimation converges.At each iteration step, IMRP perform pleiotropy test to update which genetic instrument variants showing pleiotropy evidence $(P<0.05)$ by performing the test $T_{\text {Pleio }}=\frac{\dot{\Gamma} \quad \beta \hat{\gamma}}{\text { ivar }(\hat{\mathbf{\Gamma}}-\beta \hat{\gamma})}$, where $\hat{\Gamma}$ and $\hat{\gamma}$ are the estimated effect sizes of an IV on the exposure and the outcome, respectively, and $\beta$ is the causal estimate which is updated at each iterative step. IMRP takes the advantages of MR-Egger, which is less bias, and IVW, which is efficient. IMRP can be applied to GWAS summary statistics of an exposure and an outcome obtained with overlapped or non-overlapped samples. To ensure the causal estimate is robust, we also applied a substantially different MR approach MRmix ${ }^{23}$, which is an estimating equation approach that assumes $\hat{\Gamma}-\beta \hat{\gamma}$ follow a normal mixture model.MRmix usually shows a good trade-off between bias and variance even with more than $50 \%$ invalid IVs ${ }^{23}$.

GWAS of pleiotropy analysis for SBP and DBP. We first perform a bi-directional MR analysis by IMRP to estimate the causal effect using genome wide significant independent variants associated with SBP and DBP separately in UKB-ICBP summary statistics. We then perform the pleiotropy test $T_{\text {Pleio }}$ to all the

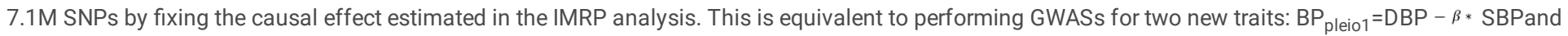
$\mathrm{BP}_{\text {pleio2 }}=\mathrm{SBP}-\beta^{\prime}$ * DBP, where $\beta$ and $\beta^{\prime}$ are the estimated causal effects of SBP on DBP and DBP on SBP, respectively. We noted BP pleio1 and BP pleio2 were highly correlated and represented essentially one phenotype $\mathrm{BP}_{\text {pleio. }}$. We declared a variant is significant when its $\mathrm{P}$-value is less than $5 \times 10^{8}$. In the replication analysis in using MVP summary statistics, we applied the causal effect estimate obtained in UKB-ICBP data. Thus, the replication analysis had the same analytic model as in the UKB-ICBP.

Genomic inflation and confounding. We applied the LD score regression method ${ }^{24}$ to test for genomic inflation in the GWAS pleiotropy analysis. It is expected that $\mathrm{BP}_{\text {pleio }}$ will have large genomic control inflation coefficient because of large sample sizes and dense genetic variants in high LD43. The GC lambda was 1.533 and LDSR intercept was 1.057 (0.013), with inflation ratio $4.23 \%$, suggesting little inflation in the pleiotropic analysis.

Novel locus definition. Novel loci were defined as the variants reaching genome wide significance and are $1 \mathrm{Mb}$ away from known BP variants as well as LD $\mathrm{r}^{2}$ $<0.05$ with any known BP variants. Novel signals at known loci are variants within $1 \mathrm{Mb}$ region of known BP variants and reach genome-wide significance level, as well as not being in LD with any known BP variants $\left(r^{2}<0.05\right)$. The $1000 \mathrm{G}$ European ancestry data was used as the reference genetic data for LD calculation.

Functional analyses. We evaluated all sentinel SNPs at novel loci for evidence of mediation of expression quantitative trait loci (eQTL) and alternative splicing quantitative trait loci (sQTL) in all 44 tissues using the Genotype-Tissue Expression (GTEx) database. Following the method in Evangelou et al. ${ }^{6}$, a locus is annotated with a given eGene(sGene) only if the most significant eQTL(sQTL) SNP for the given eGene(sGene) is in high LD ( $\left.r^{2} \geq 0.8\right)$ with the sentinel SNP. We performed overall enrichment testing but using the mediation and pleiotropic variants separately. We used DEPICT ${ }^{27}$ (Data-driven Expression Prioritized Integration for Complex Traits) to identify tissues and cells that are highly expressed at genes within the BP mediation loci, as well as BP pleiotropic loci. We also used DEPICT to test for enrichment in gene sets associated with biological annotations including GO ontology, mouse knockout phenotype studies and protein-protein interaction subnetworks. We reported significant enrichments with a false discovery rate $<0.05$. Analysis was done using the platform: Complex-Traits Genetics Virtual Lab ${ }^{44}$

Genetic risk score (GRS) and pleiotropic Genetic risk scores (PGRS). We constructed a traditional genetic risk score using independent 1,616 genome wide significant BP variants from UKB-ICBP. We first constructed SBP and DBP weighted GRSs and then derived a single BP GRS as the average of SBP and DBP GRSs. This approach was used in the literature ${ }^{6}$ to estimate the combined effect of the BP variants on BP and risks of hypertension and CVD. In addition, we constructed a pleiotropic genetic risk score PGRS using the 906 pleiotropic variants in a similar way. We first constructed $\mathrm{BP}_{\text {pleio1 }}$ and $\mathrm{BP}_{\text {pleio2 }}$ weighted PGRSs and next derived a single PGRS as the difference of SBP and DBP GRSs. The composite GRS was constructed by joint model of GRS and PGRS in a linear regression. We also performed a linear regression by jointly modeling GRS and PGRS, as well as the interaction of age*GRS and age*PGRS on blood pressure in the UKB data. Similarly, we performed logistic regression of GRS, PGRS, age*GRS and age*PGRS on risk of hypertension and cardiovascular events at baseline in the UKB data. We examined whether PGRS is able to predict additional variations of BP, hypertension and CVD after accounting for GRS. We also examined the age-varying effects of GRS and PGRS by testing the interaction effects. Our analysis included 386,752 unrelated individuals of European ancestry with phenotypes measured at baseline, respectively. To assess the association of GRS, PGRS and their interactions with age on BP, risk of hypertension and CVD, we performed the regression analysis, with adjustment for sex, age, BMI, geographical region and 10 genetic principal components. CVD was defined in unrelated participants in UKB data on the basis of self-reported medical history and linkage to hospitalization and mortality data ${ }^{6}$.

We assessed the association of the GRS, PGRS and their interactions with age on blood pressure in unrelated Africans ( $n=7,904)$ and South Asians ( $n=$ 8,509) from the UKB to see whether blood pressure-associated SNPs identified from GWAS predominantly in Europeans are also associated with blood pressure in populations of non-European ancestry. All the analyses were performed using the residuals after adjusting for sex, age, BMI, geographical region and 10 genetic principal components.

Cross-trait lookups of novel loci: We supplied the index SNPs at the novel loci observed in UK Biobank-ICBP pleiotropic analyses to FUMA ${ }^{26}$ and GWAS catalog ${ }^{25}$ to investigate the trait pleiotropy with traits other than BP, extracting all association results with P $<5 \times 10-8$, for all SNPs in high LD ( $r 2 \geq 0.8$ ).

\section{Declarations}

\section{URLs}

CTG-View: https://view.genoma.io/ 
Depict: https://data.broadinstitute.org/mpg/depict/

GTEx: www.gtexportal.org

FUMA: https://fuma.ctglab.nl/

IMRP: https://github.com/XiaofengZhuCase/IMRP

MRmix://github.com/gqi/MRMix

\section{Data Availability}

Full summary statistics related to UKB-ICBP were obtained through request to the authors of UKB-ICBP Paul Elliott or Mark Caulfield. Summary statistics relating to the Million Veteran Program (MVP) are publically available with the accession code phs001672.v1.p1:

https://www.ncbi.nlm.nih.gov/projects/gap/cgi-bin/study.cgi?study_id=phs001672.v1.p1. The UK BioBank data are available upon application to the UKBiobank (https://www.ukbiobank.ac.uk). The coronary artery disease and myocardial infraction summary statistics can be downloaded at http://www.cardiogramplusc4d.org/data-downloads/; Stroke summary statistics can be downloaded at: http://megastroke.org/privacy.html.

\section{Acknowledgements}

This work was supported by grant HG011052 (to XZ) from the National Human Genome Research Institute (NHGRI) and HL086694 (A.C.) from the National Heart, Lung and Blood Institute. We thank Dr. Jacklyn Hellwege for providing the list of published BP loci.

Data on coronary artery disease / myocardial infarction have been contributed by CARDIoGRAMplusC4D investigators and have been downloaded from wWw.CARDIOGRAMPLUSC4D.ORG'. For the Exome chip study please acknowledge the source of the data as follows: 'Data on coronary artery disease / myocardial infarction have been contributed by the Myocardial Infarction Genetics and CARDloGRAM Exome investigators and have been downloaded from WWW.CARDIOGRAMPLUSC4D.ORG

The MEGASTROKE project received funding from sources specified at http://www.megastroke.org/acknowledgments.html

\section{Author contributions}

X.Z conceived and designed the study. X.Z, L.Z. and H.W. performed analysis. X.Z. drafted the initial manuscript. All authors critically revised and approved the manuscript.

\section{Competing interests}

There is no competing interest.

\section{References}

1. Rapsomaniki, E., Timmis, A., George, J., Pujades-Rodriguez, M., Shah, A.D., Denaxas, S., White, I.R., Caulfield, M.J., Deanfield, J.E., Smeeth, L., et al. (2014). Blood pressure and incidence of twelve cardiovascular diseases: lifetime risks, healthy life-years lost, and age-specific associations in 1.25 million people. Lancet 383, 1899-1911.

2. Ehret, G.B., Ferreira, T., Chasman, D.I., Jackson, A.U., Schmidt, E.M., Johnson, T., Thorleifsson, G., Luan, J., Donnelly, L.A., Kanoni, S., et al. (2016). The genetics of blood pressure regulation and its target organs from association studies in 342,415 individuals. Nat Genet 48, $1171-1184$.

3. Ehret, G.B., Munroe, P.B., Rice, K.M., Bochud, M., Johnson, A.D., Chasman, D.I., Smith, A.V., Tobin, M.D., Verwoert, G.C., Hwang, S.J., et al. (2011). Genetic variants in novel pathways influence blood pressure and cardiovascular disease risk. Nature 478, 103-109.

4. Hoffmann, T.J., Theusch, E., Haldar, T., Ranatunga, D.K., Jorgenson, E., Medina, M.W., Kvale, M.N., Kwok, P.Y., Schaefer, C., Krauss, R.M., et al. (2018). A large electronic-health-record-based genome-wide study of serum lipids. Nat Genet 50, 401-413.

5. Warren, H.R., Evangelou, E., Cabrera, C.P., Gao, H., Ren, M., Mifsud, B., Ntalla, I., Surendran, P., Liu, C., Cook, J.P., et al. (2017). Genome-wide association analysis identifies novel blood pressure loci and offers biological insights into cardiovascular risk. Nat Genet.

6. Evangelou, E., Warren, H.R., Mosen-Ansorena, D., Mifsud, B., Pazoki, R., Gao, H., Ntritsos, G., Dimou, N., Cabrera, C.P., Karaman, I., et al. (2018). Genetic analysis of over 1 million people identifies 535 new loci associated with blood pressure traits. Nat Genet 50, 1412-1425.

7. Franceschini, N., Fox, E., Zhang, Z., Edwards, T.L., Nalls, M.A., Sung, Y.J., Tayo, B.O., Sun, Y.V., Gottesman, O., Adeyemo, A., et al. (2013). Genome-wide association analysis of blood-pressure traits in African-ancestry individuals reveals common associated genes in African and non-African populations. Am J Hum Genet 93, 545-554.

8. Liang, J., Le, T.H., Edwards, D.R.V., Tayo, B.O., Gaulton, K.J., Smith, J.A., Lu, Y., Jensen, R.A., Chen, G., Yanek, L.R., et al. (2017). Single-trait and multi-trait genome-wide association analyses identify novel loci for blood pressure in African-ancestry populations. PLoS Genet 13, e1006728.

9. Zhu, X., Feng, T., Tayo, B.O., Liang, J., Young, J.H., Franceschini, N., Smith, J.A., Yanek, L.R., Sun, Y.V., Edwards, T.L., et al. (2015). Meta-analysis of correlated traits via summary statistics from GWASs with an application in hypertension. Am J Hum Genet 96, 21-36.

10. Liu, C., Kraja, A.T., Smith, J.A., Brody, J.A., Franceschini, N., Bis, J.C., Rice, K., Morrison, A.C., Lu, Y., Weiss, S., et al. (2016). Meta-analysis identifies common and rare variants influencing blood pressure and overlapping with metabolic trait loci. Nat Genet 48, 1162-1170. 
11. Surendran, P., Drenos, F., Young, R., Warren, H., Cook, J.P., Manning, A.K., Grarup, N., Sim, X., Barnes, D.R., Witkowska, K., et al. (2016). Trans-ancestry metaanalyses identify rare and common variants associated with blood pressure and hypertension. Nat Genet 48, 1151-1161.

12. Giri, A., Hellwege, J.N., Keaton, J.M., Park, J., Qiu, C., Warren, H.R., Torstenson, E.S., Kovesdy, C.P., Sun, Y.V., Wilson, O.D., et al. (2019). Trans-ethnic association study of blood pressure determinants in over 750,000 individuals. Nat Genet 51, 51-62.

13. Sung, Y.J., Winkler, T.W., de Las Fuentes, L., Bentley, A.R., Brown, M.R., Kraja, A.T., Schwander, K., Ntalla, I., Guo, X., Franceschini, N., et al. (2018). A LargeScale Multi-ancestry Genome-wide Study Accounting for Smoking Behavior Identifies Multiple Significant Loci for Blood Pressure. Am J Hum Genet 102, 375-400.

14. Sung, Y.J., de Las Fuentes, L., Winkler, T.W., Chasman, D.I., Bentley, A.R., Kraja, A.T., Ntalla, I., Warren, H.R., Guo, X., Schwander, K., et al. (2019). A multiancestry genome-wide study incorporating gene-smoking interactions identifies multiple new loci for pulse pressure and mean arterial pressure. Hum Mol Genet.

15. Schillaci, G., and Pucci, G. (2010). The dynamic relationship between systolic and diastolic blood pressure: yet another marker of vascular aging? Hypertension research : official journal of the Japanese Society of Hypertension 33, 659-661.

16. Simino, J., Shi, G., Bis, J.C., Chasman, D.I., Ehret, G.B., Gu, X., Guo, X., Hwang, S.J., Sijbrands, E., Smith, A.V., et al. (2014). Gene-age interactions in blood pressure regulation: a large-scale investigation with the CHARGE, Global BPgen, and ICBP Consortia. Am J Hum Genet 95, 24-38.

17. Shi, G., Gu, C.C., Kraja, A.T., Arnett, D.K., Myers, R.H., Pankow, J.S., Hunt, S.C., and Rao, D.C. (2009). Genetic effect on blood pressure is modulated by age: the Hypertension Genetic Epidemiology Network Study. Hypertension 53, 35-41.

18. Dumitrescu, L., Brown-Gentry, K., Goodloe, R., Glenn, K., Yang, W., Kornegay, N., Pui, C.H., Relling, M.V., and Crawford, D.C. (2011). Evidence for age as a modifier of genetic associations for lipid levels. Ann Hum Genet 75, 589-597.

19. Lasky-Su, J., Lyon, H.N., Emilsson, V., Heid, I.M., Molony, C., Raby, B.A., Lazarus, R., Klanderman, B., Soto-Quiros, M.E., Avila, L., et al. (2008). On the replication of genetic associations: timing can be everything! Am J Hum Genet 82, 849-858.

20. Jiang, X., Holmes, C., and McVean, G. (2020). Age modulates genetic risk for multiple common diseases. bioRxiv.

21. Smith, G.D., and Ebrahim, S. (2003). 'Mendelian randomization': can genetic epidemiology contribute to understanding environmental determinants of disease? International journal of epidemiology 32, 1-22.

22. Zhu, X., Li, X., Xu, R., and Wang, T. (2020). An iterative approach to detect pleiotropy and perform mendelian randomization analysis using GWAS summary statistics. Bioinformatics (Oxford, England).

23. Qi, G., and Chatterjee, N. (2019). Mendelian randomization analysis using mixture models for robust and efficient estimation of causal effects. Nature communications 10, 1941.

24. Bulik-Sullivan, B.K., Loh, P.R., Finucane, H.K., Ripke, S., Yang, J., Schizophrenia Working Group of the Psychiatric Genomics, C., Patterson, N., Daly, M.J., Price, A.L., and Neale, B.M. (2015). LD Score regression distinguishes confounding from polygenicity in genome-wide association studies. Nat Genet 47, 291-295.

25. MacArthur, J., Bowler, E., Cerezo, M., Gil, L., Hall, P., Hastings, E., Junkins, H., McMahon, A., Milano, A., Morales, J., et al. (2017). The new NHGRI-EBI Catalog of published genome-wide association studies (GWAS Catalog). Nucleic acids research 45, D896-D901.

26. Watanabe, K., Taskesen, E., van Bochoven, A., and Posthuma, D. (2017). Functional mapping and annotation of genetic associations with FUMA. Nature communications 8, 1826.

27. Pers, T.H., Karjalainen, J.M., Chan, Y., Westra, H.J., Wood, A.R., Yang, J., Lui, J.C., Vedantam, S., Gustafsson, S., Esko, T., et al. (2015). Biological interpretation of genome-wide association studies using predicted gene functions. Nature communications 6, 5890.

28. Inouye, M., Abraham, G., Nelson, C.P., Wood, A.M., Sweeting, M.J., Dudbridge, F., Lai, F.Y., Kaptoge, S., Brozynska, M., Wang, T., et al. (2018). Genomic Risk Prediction of Coronary Artery Disease in 480,000 Adults: Implications for Primary Prevention. Journal of the American College of Cardiology 72, 18831893.

29. Maier, R.M., Zhu, Z., Lee, S.H., Trzaskowski, M., Ruderfer, D.M., Stahl, E.A., Ripke, S., Wray, N.R., Yang, J., Visscher, P.M., et al. (2018). Improving genetic prediction by leveraging genetic correlations among human diseases and traits. Nature communications 9, 989.

30. Krapohl, E., Patel, H., Newhouse, S., Curtis, C.J., von Stumm, S., Dale, P.S., Zabaneh, D., Breen, G., O'Reilly, P.F., and Plomin, R. (2018). Multi-polygenic score approach to trait prediction. Mol Psychiatry 23, 1368-1374.

31. Richardson, T.G., Harrison, S., Hemani, G., and Davey Smith, G. (2019). An atlas of polygenic risk score associations to highlight putative causal relationships across the human phenome. eLife 8.

32. Franklin, S.S. (2004). Pulse pressure as a risk factor. Clinical and experimental hypertension 26, 645-652.

33. Khan, S.S., Cooper, R., and Greenland, P. (2020). Do Polygenic Risk Scores Improve Patient Selection for Prevention of Coronary Artery Disease? JAMA 323, 614-615.

34. Elliott, J., Bodinier, B., Bond, T.A., Chadeau-Hyam, M., Evangelou, E., Moons, K.G.M., Dehghan, A., Muller, D.C., Elliott, P., and Tzoulaki, I. (2020). Predictive Accuracy of a Polygenic Risk Score-Enhanced Prediction Model vs a Clinical Risk Score for Coronary Artery Disease. JAMA 323, $636-645$.

35. Mosley, J.D., Gupta, D.K., Tan, J., Yao, J., Wells, Q.S., Shaffer, C.M., Kundu, S., Robinson-Cohen, C., Psaty, B.M., Rich, S.S., et al. (2020). Predictive Accuracy of a Polygenic Risk Score Compared With a Clinical Risk Score for Incident Coronary Heart Disease. JAMA 323, 627-635.

36. Franklin, S.S. (1999). Ageing and hypertension: the assessment of blood pressure indices in predicting coronary heart disease. Journal of hypertension Supplement : official journal of the International Society of Hypertension 17, S29-36.

37. Boyle, E.A., Li, Y.I., and Pritchard, J.K. (2017). An Expanded View of Complex Traits: From Polygenic to Omnigenic. Cell 169, $1177-1186$.

38. Liu, X., Li, Y.I., and Pritchard, J.K. (2019). Trans Effects on Gene Expression Can Drive Omnigenic Inheritance. Cell 177, $1022-1034$ e1026.

Page $7 / 11$ 
39. Chakravarti, A., and Turner, T.N. (2016). Revealing rate-limiting steps in complex disease biology: The crucial importance of studying rare, extremephenotype families. BioEssays : news and reviews in molecular, cellular and developmental biology 38, 578-586.

40. Egger, M., Davey Smith, G., Schneider, M., and Minder, C. (1997). Bias in meta-analysis detected by a simple, graphical test. Bmj 315, 629-634.

41. Borenstein, M., Hedges, L., Higgins, J., Rothstein, H. . (2009). Generality of the basic inverse-variance method. In: Introduction to Meta-analysis. Chichester, UK: Wiley.

42. Burgess, S., Butterworth, A., and Thompson, S.G. (2013). Mendelian randomization analysis with multiple genetic variants using summarized data. Genet Epidemiol 37, 658-665.

43. Marouli, E., Graff, M., Medina-Gomez, C., Lo, K.S., Wood, A.R., Kjaer, T.R., Fine, R.S., Lu, Y., Schurmann, C., Highland, H.M., et al. (2017). Rare and lowfrequency coding variants alter human adult height. Nature 542, 186-190.

44. Cuellar-Partida, G., Lundberg, M., Kho, P.F., D’Urso, S., Gutierrez-Mondragon, L., and Hwang, L. (2019). Complex-Traits Genetics Virtual Lab: A communitydriven web platform for post-GWAS analyses. bioRxiv.

\section{Tables}

Table 1. The 17 novel BP loci identified by pleiotropic analysis

\begin{tabular}{|c|c|c|c|c|c|c|c|c|}
\hline SNP & CHR & $\mathrm{BP}$ & P_SBP & P_DBP & P_PP & $\mathrm{BP}_{\text {pleio }}$ & MVP $\mathrm{P}_{\text {pleio }}$ & Annotated genes \\
\hline rs11162906 & 1 & 80500074 & $3.19 \times 10^{-2}$ & $1.75 \times 10^{-2}$ & $1.78 \times 10^{-6}$ & $2.41 \times 10^{-9}$ & $1.43 \times 10^{-3}$ & AC098657.2 \\
\hline rs17713879 & 2 & 254215 & $5.19 \times 10^{-2}$ & $2.66 \times 10^{-2}$ & $2.05 \times 10^{-5}$ & $3.75 \times 10^{-8}$ & $7.97 \times 10^{-4}$ & SH3YL1 \\
\hline rs6735304 & 2 & 101617631 & $4.18 \times 10^{-2}$ & $2.38 \times 10^{-2}$ & $1.43 \times 10^{-6}$ & $1.47 \times 10^{-8}$ & $9.31 \times 10^{-4}$ & RPL31/TBC1D8 \\
\hline rs12470661 & 2 & 232060050 & $5.09 \times 10^{-2}$ & $3.07 \times 10^{-3}$ & $6.68 \times 10^{-7}$ & $5.33 \times 10^{-11}$ & $1.25 \times 10^{-3}$ & HTR2B/ARMC9 \\
\hline rs10947978 & 6 & 41471608 & $7.01 \times 10^{-1}$ & $9.89 \times 10^{-6}$ & $1.44 \times 10^{-5}$ & $2.65 \times 10^{-11}$ & $1.81 \times 10^{-2}$ & LINC01276 \\
\hline rs56098119 & 6 & 90296727 & $7.63 \times 10^{-2}$ & $1.38 \times 10^{-2}$ & $6.81 \times 10^{-6}$ & $1.69 \times 10^{-8}$ & $3.18 \times 10^{-2}$ & ANKRD6 \\
\hline rs150953973 & 6 & 120780033 & $2.98 \times 10^{-1}$ & $9.7 \times 10^{-4}$ & $4.01 \times 10^{-6}$ & $3.54 \times 10^{-9}$ & $2.07 \times 10^{-2}$ & RNU6-2 \\
\hline rs180271 & 7 & 93539479 & $6.11 \times 10^{-1}$ & $1.72 \times 10^{-4}$ & $2.63 \times 10^{-5}$ & $3.69 \times 10^{-9}$ & $5.64 \times 10^{-3}$ & GNGT1 \\
\hline rs11989271 & 8 & 122632611 & $1.51 \times 10^{-1}$ & $8.47 \times 10^{-4}$ & $7.80 \times 10^{-7}$ & $1.14 \times 10^{-10}$ & $7.38 \times 10^{-3}$ & HAS2 \\
\hline rs10868842 & 9 & 73119085 & $1.57 \times 10^{-1}$ & $3.58 \times 10^{-4}$ & $9.71 \times 10^{-6}$ & $1.37 \times 10^{-11}$ & $2.16 \times 10^{-2}$ & LINC00583 \\
\hline rs12768143 & 10 & 22808844 & $3.92 \times 10^{-2}$ & $5.03 \times 10^{-3}$ & $8.61 \times 10^{-8}$ & $9.18 \times 10^{-11}$ & $1.61 \times 10^{-2}$ & PIP4K2A \\
\hline rs1343676 & 12 & 33537387 & $7.19 \times 10^{-1}$ & $2.03 \times 10^{-7}$ & $8.65 \times 10^{-7}$ & $9.56 \times 10^{-15}$ & $1.26 \times 10^{-2}$ & SYT10 \\
\hline rs7322054 & 13 & 38246708 & $6.71 \times 10^{-1}$ & $1.99 \times 10^{-7}$ & $7.76 \times 10^{-4}$ & $1.04 \times 10^{-11}$ & $8.10 \times 10^{-3}$ & TRPC4 \\
\hline rs61972411 & 13 & 100602630 & $2.82 \times 10^{-4}$ & $6.59 \times 10^{-1}$ & $1.87 \times 10^{-7}$ & $1.45 \times 10^{-8}$ & $2.29 \times 10^{-2}$ & LOC101927437 \\
\hline rs62621400 & 15 & 101718239 & $2.25 \times 10^{-1}$ & $1.70 \times 10^{-3}$ & $1.94 \times 10^{-5}$ & $3.76 \times 10^{-9}$ & $2.12 \times 10^{-3}$ & CHSY1 \\
\hline rs116643984 & 15 & 101791212 & $1.64 \times 10^{-1}$ & $7.73 \times 10^{-5}$ & $5.14 \times 10^{-8}$ & $4.04 \times 10^{-13}$ & $1.04 \times 10^{-2}$ & CHSY1 \\
\hline rs73937040 & 18 & 3258733 & $7.08 \times 10^{-2}$ & $2.07 \times 10^{-2}$ & $7.77 \times 10^{-6}$ & $4.67 \times 10^{-8}$ & $2.01 \times 10^{-6}$ & MYL12A/MYL12B \\
\hline rs146827176 & 20 & 35169916 & $6.48 \times 10^{-1}$ & $3.10 \times 10^{-6}$ & $1.01 \times 10^{-3}$ & $2.13 \times 10^{-9}$ & $9.76 \times 10^{-3}$ & LOC101926987|MYL9 \\
\hline
\end{tabular}

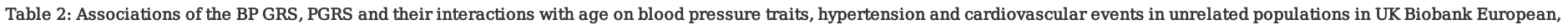




\begin{tabular}{|c|c|c|c|c|c|c|c|c|c|c|c|c|c|c|c|c|}
\hline \\
\hline & \multicolumn{3}{|c|}{ GRS } & \multicolumn{3}{|c|}{ PGRS } & \multicolumn{3}{|c|}{ Heritability $^{c}$} & \multicolumn{3}{|c|}{ Age*GRS } & \multicolumn{3}{|c|}{ Age*PGRS } & \multirow[b]{2}{*}{ I } \\
\hline Trait & Effect $^{\mathrm{a}}$ & $95 \% \mathrm{CI}$ & $P$ & Effect $^{a}$ & $95 \% \mathrm{CI}$ & $P$ & $\begin{array}{l}\text { GRS } \\
\text { only } \\
\text { (\%) }\end{array}$ & $\begin{array}{c}\text { GRS+ } \\
\text { PGRS } \\
(\%) \\
\end{array}$ & $\begin{array}{c}\text { fold } \\
\text { change } \\
(\%)\end{array}$ & Effect $^{a}$ & $95 \% \mathrm{CI}$ & $P$ & Effect ${ }^{a}$ & $95 \% \mathrm{CI}$ & $\mathrm{P}$ & \\
\hline SBP & 4.56 & $(4.51 ; 4.62)$ & $<10^{-300}$ & 2.01 & $(1.96 ; 2.07)$ & $<10^{-300}$ & 5.91 & 7.13 & 20.64 & 0.011 & $(0.004 ; 0.018)$ & $2.02 \times 10^{-3}$ & 0.068 & $(0.014 ; 0.123)$ & $1.32 \times 10^{-2}$ & $\overline{385}$, \\
\hline DBP & 2.44 & $(2.41 ; 2.47)$ & $<10^{-300}$ & -0.83 & $(-0.86 ;-0.80)$ & $<10^{-300}$ & 6.09 & 6.75 & 10.83 & -0.026 & $(-0.030 ;-0.022)$ & $1.41 \times 10^{-39}$ & -0.101 & $(-0.132 ;-0.071)$ & $8.40 \times 10^{-11}$ & 385 , \\
\hline $\mathrm{PP}$ & 2.12 & $(2.09 ; 2.16)$ & $<10^{-300}$ & 2.84 & $(2.80 ; 2.88)$ & $<10^{-300}$ & 2.23 & 7.27 & 226.65 & 0.037 & $\begin{array}{l}(0.032 ; 0.042) \\
\end{array}$ & $1.40 \times 10^{-51}$ & 0.170 & $(0.132 ; 0.208)$ & $1.05 \times 10^{-18}$ & 385 , \\
\hline HTN & 1.64 & $(1.63 ; 1.66)$ & $<10^{-300}$ & 1.15 & $(1.14 ; 1.16)$ & $<10^{-300}$ & 4.71 & 5.14 & 1 & 0.997 & (0.996;0.998) & $1.50 \times 10^{-11}$ & 1.005 & $(1.004 ; 1.006)$ & $7.74 \times 10^{-29}$ & 385, \\
\hline CVD & 1.21 & $(1.20 ; 1.23)$ & $1.71 \times 10^{-231}$ & 1.06 & $(1.05 ; 1.08)$ & $2.74 \times 10^{-25}$ & 0.47 & 0.53 & 14.1 & 0.999 & $\begin{array}{l}0.997 ; 1.001) \\
\end{array}$ & $4.25 \times 10^{-1}$ & 1.002 & $(1.00 ; 1.004)$ & $3.0 \times 10^{-2}$ & 385, \\
\hline $\mathrm{CVD}^{\mathrm{b}}$ & 1.66 & $(1.58 ; 1.74)$ & $9.83 \times 10^{-97}$ & 1.19 & $(1.14 ; 1.25)$ & $1.28 \times 10^{-13}$ & 0.74 & 0.89 & 20.3 & 1.002 & $(0.995 ; 1.009)$ & $6.14 \times 10^{-1}$ & 1.004 & $(0.996 ; 1.011)$ & $3.37 \times 10^{-1}$ & $96, \mathrm{9}$ \\
\hline \multicolumn{17}{|c|}{ B. Africans } \\
\hline SBP & 2.71 & $(2.28 ; 3.13)$ & $1.45 \times 10^{-39}$ & 1.21 & $(0.77 ; 1.65)$ & $8.34 \times 10^{-8}$ & 1.74 & 1.91 & 9.91 & 0.010 & $(-0.041 ; 0.061)$ & $7.0 \times 10^{-1}$ & 0.045 & $(-0.007 ; 0.096)$ & $9.02 \times 10^{-1}$ & $\overline{7,8}$ \\
\hline DBP & 1.53 & $(1.27 ; 1.79)$ & $4.19 \times 10^{-31}$ & -0.35 & $(-0.62 ;-0.08)$ & $1.09 \times 10^{-2}$ & 1.59 & 1.73 & 8.72 & -0.015 & $(-0.046 ; 0.016)$ & $3.36 \times 10^{-1}$ & 0.002 & $(-0.029 ; 0.033)$ & $9.0 \times 10^{-1}$ & $\overline{7,8}$ \\
\hline PP & 1.18 & $(0.91 ; 1.45)$ & $1.61 \times 10^{-17}$ & 1.56 & $(1.28 ; 1.84)$ & $2.05 \times 10^{-27}$ & 0.75 & 1.83 & 143.86 & 0.025 & $(-0.007 ; 0.057)$ & $1.28 \times 10^{-1}$ & 0.043 & $(0.010 ; 0.075)$ & $1.1 \times 10^{-2}$ & $\overline{7,8}$ \\
\hline HTN & 1.32 & $(1.25 ; 1.39)$ & $7.17 \times 10^{-27}$ & 1.04 & $(0.99 ; 1.09)$ & $1.54 \times 10^{-1}$ & 0.89 & 1.11 & 22.8 & 1.001 & $(0.994 ; 1.007)$ & $8.26 \times 10^{-1}$ & 1.005 & $\begin{array}{l}0.999 ; 1.012) \\
\end{array}$ & $1.2 \times 10^{-1}$ & $\overline{7,8}$ \\
\hline CVD & 1.14 & $(1.03 ; 1.26)$ & $9.88 \times 10^{-3}$ & 1.07 & $(0.97 ; 1.19)$ & $1.85 \times 10^{-1}$ & 0.18 & 0.22 & 21.9 & 1.003 & $(0.992 ; 1.014)$ & $6.04 \times 10^{-1}$ & 1.000 & (0.989;1.012) & 1.0 & $\overline{7,8}$ \\
\hline \multicolumn{17}{|c|}{ C. Asians } \\
\hline SBP & 2.92 & $(2.54 ; 3.30)$ & $2.71 \times 10^{-50}$ & 1.25 & $(0.86 ; 1.64)$ & $3.21 \times 10^{-10}$ & 2.53 & 3.00 & 18.48 & -0.011 & $(-0.056 ; 0.035)$ & $6.49 \times 10^{-1}$ & 0.015 & $(-0.030 ; 0.060)$ & $5.12 \times 10^{-1}$ & $\overline{8,3}$ \\
\hline DBP & 1.69 & $(1.47 ; 1.91)$ & $5.95 \times 10^{-51}$ & -0.64 & $(-0.87 ;-0.42)$ & $1.69 \times 10^{-8}$ & 2.58 & 2.89 & 12.16 & -0.017 & $(-0.043 ; 0.009)$ & $1.96 \times 10^{-1}$ & -0.047 & $(-0.073 ;-0.021)$ & $3.64 \times 10^{-4}$ & $\overline{8,3}$ \\
\hline PP & 1.23 & $(0.97 ; 1.48)$ & $3.85 \times 10^{-21}$ & 1.89 & $(1.63 ; 2.15)$ & $1.50 \times 10^{-46}$ & 1.00 & 3.33 & 233.47 & 0.007 & $(-0.023 ; 0.037)$ & $6.65 \times 10^{-1}$ & 0.062 & $(0.032 ; 0.092)$ & $4.71 \times 10^{-5}$ & $\overline{8,3}$ \\
\hline HTN & 1.37 & $(1.30 ; 1.44)$ & $3.90 \times 10^{-36}$ & 1.10 & $(1.05 ; 1.16)$ & $9.27 \times 10^{-5}$ & 1.84 & 2.08 & 13.4 & 0.998 & $(0.992 ; 1.004)$ & $5.23 \times 10^{-1}$ & 1.006 & $(1.00 ; 1.012)$ & $3.86 \times 10^{-2}$ & $\overline{8,3}$ \\
\hline CVD & 1.18 & $(1.10 ; 1.26)$ & $2.87 \times 10^{-6}$ & 1.08 & $(1.01 ; 1.16)$ & $2.81 \times 10^{-2}$ & 0.19 & 0.39 & 101. & 1.003 & $(0.995 ; 1.012)$ & $4.50 \times 10^{-1}$ & 0.991 & $(0.983 ; 1.0)$ & $4.27 \times 10^{-2}$ & $\overline{8,3}$ \\
\hline
\end{tabular}

Sex, age, BMI, 10 PCs and geographic regions were adjusted. GRS and PGRS were jointly modeled in the regression analyses.

aThe effect represents regression coefficient for SBP, DBP and PP and represents odd ratio for HTN and CVD.

${ }^{\mathrm{b}}$ The effect for CVD represents the odd ratio when comparing top quantile with bottom quantile of GRS and PGRS.

${ }^{\mathrm{c}}$ The heritability for HTN and CVD represents the Nagelkerke's $\mathrm{R}^{2}$.

Table 3. Using mediation variants and pleiotropy variants separately in MR analysis with outcomes: CAD, MI and STROKE

\begin{tabular}{|c|c|c|c|c|c|c|c|c|c|}
\hline \multirow[b]{2}{*}{ Outcome } & \multirow[b]{2}{*}{ IV type } & \multirow[b]{2}{*}{$\#$ of $\mathrm{IVs}^{\mathrm{a}}$} & \multicolumn{4}{|c|}{ IMRP } & \multicolumn{3}{|c|}{ MRmix } \\
\hline & & & Causal effect ${ }^{\mathrm{b}}$ & $95 \% \mathrm{CI}$ & $P$ & \# of PVs ${ }^{\mathrm{c}}$ & Causal effect ${ }^{b}$ & $95 \% \mathrm{CI}$ & $P$ \\
\hline \multicolumn{10}{|c|}{ Exposure: SBP } \\
\hline CAD & mediation & 758 & 1.85 & $(1.73 ; 1.97)$ & $3.73 \times 10^{-78}$ & 103 & 1.80 & $(1.56 ; 2.09)$ & $2.68 \times 10^{-15}$ \\
\hline MI & mediation & 756 & 1.77 & $(1.65 ; 1.90)$ & $1.60 \times 10^{-57}$ & 89 & 1.72 & $(1.42 ; 2.08)$ & $3.57 \times 10^{-8}$ \\
\hline stroke & mediation & 758 & 1.66 & $(1.55 ; 1.76)$ & $1.37 \times 10^{-57}$ & 77 & 1.70 & $(1.29 ; 2.24)$ & $1.80 \times 10^{-4}$ \\
\hline CAD & pleiotropy & 730 & 1.48 & $(1.34 ; 1.63)$ & $2.64 \times 10^{-14}$ & 150 & 1.49 & $(1.06 ; 2.10)$ & 0.022 \\
\hline MI & Pleiotropy & 729 & 1.32 & $(1.18 ; 1.47)$ & $7.11 \times 10^{-7}$ & 125 & 1.43 & $(1.09 ; 1.88)$ & $9.41 \times 10^{-3}$ \\
\hline stroke & pleiotropy & 730 & 1.42 & $(1.30 ; 1.55)$ & $1.98 \times 10^{-14}$ & 92 & 1.16 & $(0.81 ; 1.67)$ & 0.417 \\
\hline \multicolumn{10}{|c|}{ Exposure: DBP } \\
\hline CAD & mediation & 774 & 1.85 & $(1.74 ; 1.97)$ & $1.16 \times 10^{-80}$ & 105 & 1.86 & $(1.60 ; 2.16)$ & $5.38 \times 10^{-16}$ \\
\hline MI & mediation & 771 & 1.85 & $(1.73 ; 1.98)$ & $3.22 \times 10^{-68}$ & 91 & 1.86 & $(1.51 ; 2.29)$ & $5.65 \times 10^{-9}$ \\
\hline STROKE & mediation & 773 & 1.67 & $(1.57 ; 1.77)$ & $2.77 \times 10^{-60}$ & 79 & 1.88 & $(1.51 ; 2.33)$ & $8.84 \times 10^{-9}$ \\
\hline CAD & pleiotropy & 730 & 1.37 & $(1.24 ; 1.50)$ & $4.63 \times 10^{-11}$ & 157 & 1.34 & $(1.02 ; 1.75)$ & 0.037 \\
\hline MI & Pleiotropy & 729 & 1.13 & $(1.02 ; 1.25)$ & 0.017 & 134 & 1.31 & $(1.05 ; 1.63)$ & 0.527 \\
\hline STROKE & pleiotropy & 730 & 1.28 & $(1.18 ; 1.39)$ & $5.07 \times 10^{-9}$ & 90 & 1.13 & $(0.80 ; 1.59)$ & 0.491 \\
\hline
\end{tabular}

a\# of IVs: number of genetic instrumental variables

${ }^{\mathrm{b}}$ causal effect represents odd ratio

'\# of PVs: number of pleiotropic variants detected by IMRP.

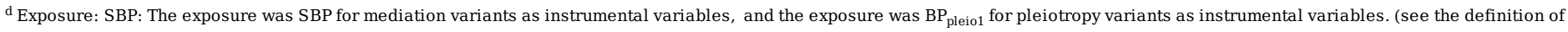

$\mathrm{BP}_{\text {pleio1 }}$ in Online Methods)

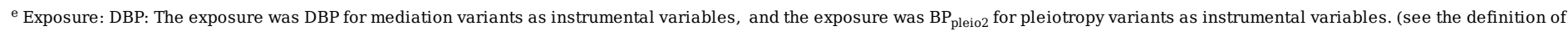

$\mathrm{BP}_{\text {pleio2 }}$ in Online Methods)

CAD: coronary artery disease

MI: myocardial infraction

\section{Figures}


A.

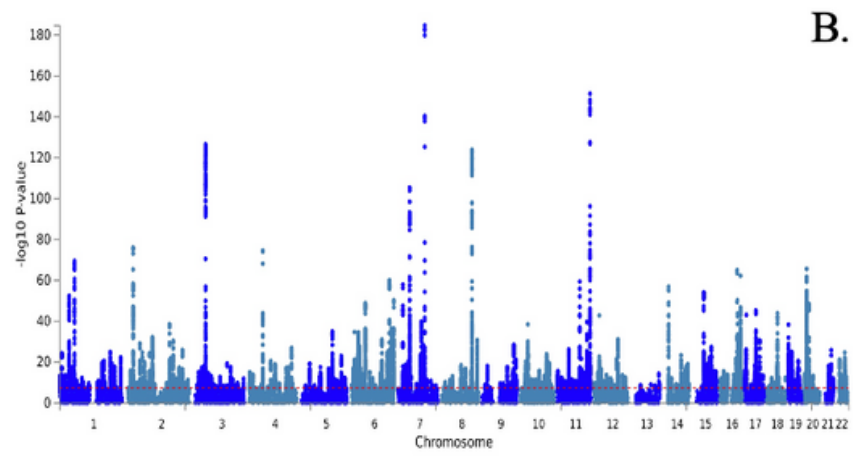

B.
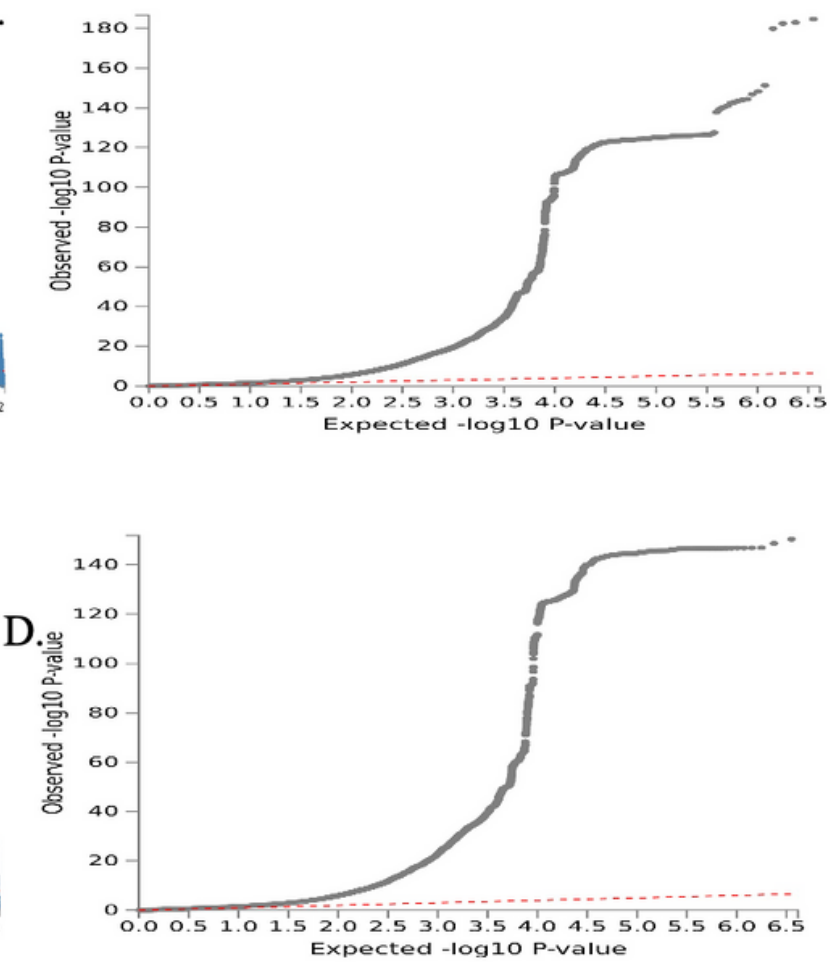

Figure 1

Manhattan and QQ plots for genome wide pleiotropy tests between SBP and DBP using UK Biobank-ICBP summary statistics. The GWAS of pleiotropy tests is equivalent to performing GWAS for two new traits: BPpleio1=DBP - $\beta^{\star}$ SBP and BPpleio2=SBP - $\beta^{\prime \prime *}$ DBP, where $\beta$ and $\beta^{\wedge}$ are the estimated causal effects of SBP on DBP and DBP on SBP, respectively. A. and B. Manhattan and QQ plots for BPpleio2. C. and D. Manhattan and QQ plots for BPpleio1. The horizontal line in Manhattans represents $\mathrm{P}-\mathrm{value}=5 \times 10-8$. The top and bottom Manhattan plots are highly similar, indicating the consistence of the two directional MR analysis. 
A.
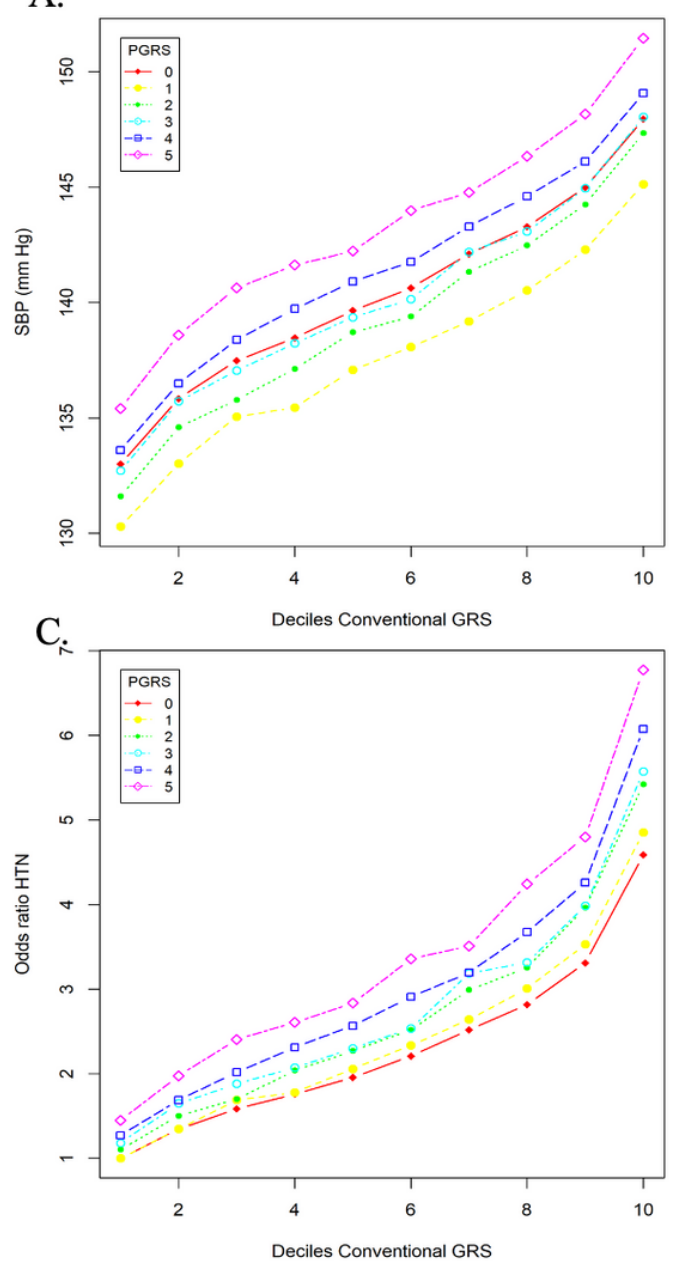

B.

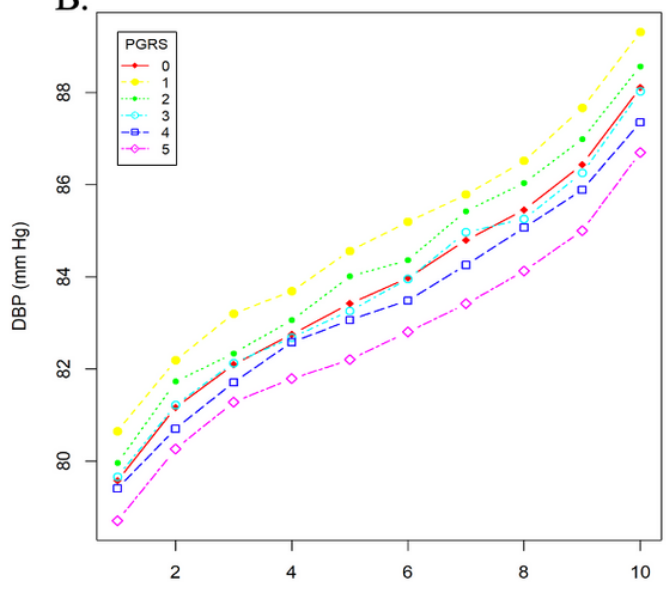

D.

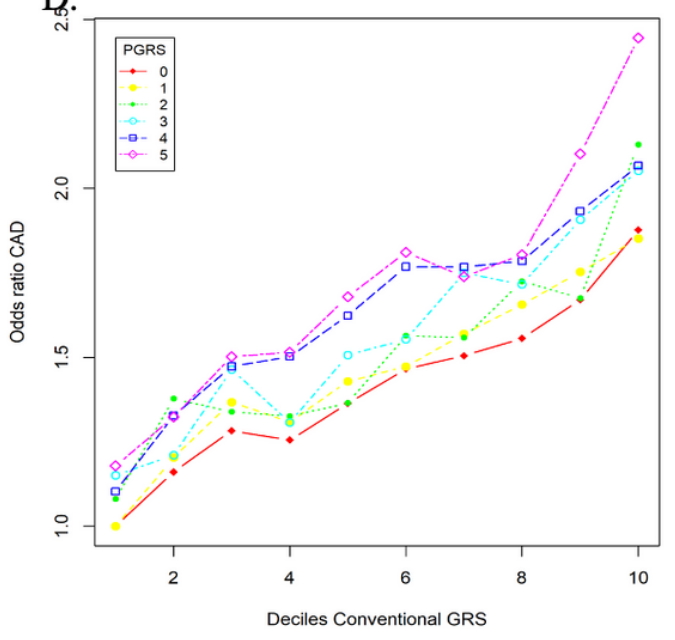

Figure 2

Relationship the joint conventional genetic risk score (GRS) and pleiotropic genetic risk score (PGRS) with blood pressure, risk of hypertension and cardiovascular disease in UK Biobank. (A) sex adjusted mean systolic blood pressure (SBP); (B) sex adjusted mean diastolic blood pressure; (C) odds ratios of hypertension (HTN) and (D) odds ratios of cardiovascular disease (CVD). GRS was calculated in every decile and PGRS was calculated every quintile. Odds ratios were calculated by comparing each of the GRS deciles and PGRS quintiles with the lowest decile and twentieth. The curves with PGRS=0 represent the models without including PGRS.

\section{Supplementary Files}

This is a list of supplementary files associated with this preprint. Click to download.

- SuppSTables.xIsx

- SuppMaterials.docx 